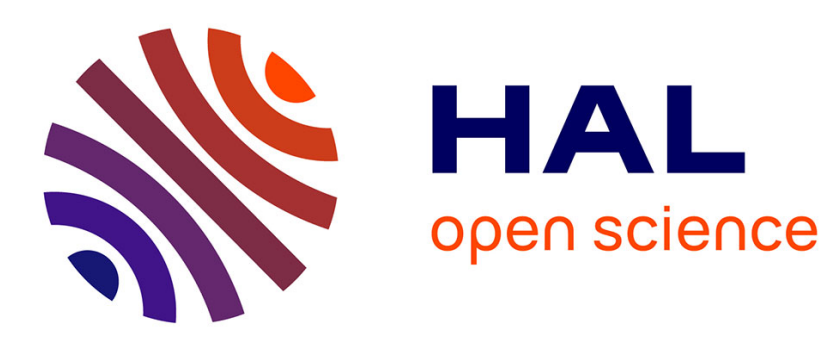

\title{
Characterization of Radio Frequency Echo using frequency sweeping and power analysis
}

\author{
Amar Zeher, Stéphane Binczak, Jerome Joli
}

\section{To cite this version:}

Amar Zeher, Stéphane Binczak, Jerome Joli. Characterization of Radio Frequency Echo using frequency sweeping and power analysis. 2014 IEEE REGION 10 TECHNICAL SYMPOSIUM, Apr 2014, Kuala Lumpur, Malaysia. pp.356-360, 10.1109/TENCONSpring.2014.6863057 . hal-01463734

\section{HAL Id: hal-01463734 \\ https://hal.science/hal-01463734}

Submitted on 17 Feb 2017

HAL is a multi-disciplinary open access archive for the deposit and dissemination of scientific research documents, whether they are published or not. The documents may come from teaching and research institutions in France or abroad, or from public or private research centers.
L'archive ouverte pluridisciplinaire HAL, est destinée au dépôt et à la diffusion de documents scientifiques de niveau recherche, publiés ou non, émanant des établissements d'enseignement et de recherche français ou étrangers, des laboratoires publics ou privés. 


\section{Characterization of Radio Frequency Echo Using Frequency Sweeping and Power Analysis}

\author{
Amar Zeher \\ LE2I CNRS UMR 6306, \\ Université de Bourgogne, \\ 9 avenue Alain Savary, BP47870 \\ 21078 Dijon cedex, France \\ Email: amar.zeher@u-bourgogne.fr
}

\author{
Stéphane Binczak \\ Laboratory LE2I CNRS UMR 6306, \\ Université de Bourgogne, \\ 9 avenue Alain Savary, BP47870 \\ 21078 Dijon cedex, France \\ Email: stbinc@u-bourgogne.fr
}

\author{
Jérome Joli \\ SELECOM, \\ ZA Alred Sauvy, \\ 66500 Prades, France. \\ Email: jjoli@selecom.fr
}

\begin{abstract}
Coupling between repeater's antennas, called Radio Frequency Echo (RFE) deteriorates signal quality and compromises system stability. If the channel insulation is insufficient, the power in the closed-loop raises with time according to the gain margin value. Multiple methods of echo cancellation exists. Generally, these techniques are based on correlation calculation and adaptive filtering. However, their efficiency and algorithm's convergence speed are calling into question under signal's modulation and real-time constraints, specially with narrow band signals having frequency hopping as GSM or TETRA. This paper describes a real time method of RFE detection and estimation independently of the nature of the incoming signal. It is based on frequency sweeping and analysis of power ripples, and it can be implemented on FPGA for real-time purpose. It gives an accurate estimation of corresponding gain margin and loop delay.

Index Terms-Radio frequency echo, gain margin, narrow band, frequency hopping.
\end{abstract}

\section{INTRODUCTION}

In regions where reception quality is poor, it is usual to locate repeaters which are deployed to expand and enhance coverage region of Base Station Transceiver (BTS) costeffectively, without the need for modulation and demodulation setups. A repeater receives signals from the main transmitter which is generally BTS on the selected carriers ; then, it retransmits them to receivers which are generally Mobile Station (MS) such as cell phones, Personal Digital Assistant (PDA) and communication terminals. The receiving signal is filtered, amplified and re-transmitted; if the repeater retransmits signals in the same frequencies, it is called Iso-Frequency Repeater (IFR), otherwise, it is called Transposing Frequency Repeater (TFR). Deploying TFR to extend coverage area is robust, but it is expensive. Contrariwise, IFR are cost-effectively and simple to set up. Nevertheless, with IFR, if the channel insulation is insufficient, a part of the transmitted signal could be picked up by the receiving antenna of the repeater, leading to RFE creation.This phenomenon is well known as the issue was first posed in acoustics field [1]. When using repeaters, Echo Cancellation is one method of compensating for external Radio Frequency (RF) coupling between antennas [2]. The echo signals must be reduced to an allowable level in order to avoid problems with distortion and oscillation [3]. Many techniques exist for cancelling echoes, notably in the area of acoustics as Wiener Filter, Linear Prediction [4] or Non-linear Acoustic Echo Cancellation Based on Volterra Filters [5]. Some of these techniques are applied to radiocommunication. Depending on the nature of concerning signal, the efficiency of these methods could be called into question. Indeed, modulation constraints as guard interval, Error Vector Magnitude (EVM), Modulation Error Ratio (MER) have to be satisfied when filtering.

Narrow band signal (NBS) with frequency hopping, as in GSM or TETRA standards, is one major difficulty when using linear filtering because of the convergence rate of adaptive algorithms which are generally too slow when compared with the hopping frequency rate and randomness [6]. Under these constraints, linear filtering increases transmission errors and creates an Adjacent-Channel Interference (ACI).

Radiofrequency signal can also be Wide Band Signal (WBS), which occupies a range of frequencies such as DVB$\mathrm{T} / \mathrm{H}(8 \mathrm{MHz}$ in France) and CDMA. NBS has a narrower band width, generally less than $1 \mathrm{MHz}$ as GSM $(200 \mathrm{KHz})$ and TETRA standards $(25 \mathrm{KHz})$. Part (a) of Fig. 1 shows an example of WBS and NBS displayed in the frequency domain without RFE.

Ripples indicates the presence of a RFE and, as illustrated in part (b) of the Fig. 1, they are only distinguishable in WBS and the noise floor parts. With noise floor or wide band, it is easily detectable and voidable by using linear adaptive algorithms.

Nevertheless, the narrower the band is, the more difficult these solutions can be used effectively. For example, adaptive filtering using Wiener solution based on the correlation calculation is a good solution when used with DVB-T or CDMA standard, but it cannot be used when GSM signal with frequency hopping is used, because of the ACI and the unwanted spurious which are created.

The transmitted signal can reach the receiving antenna by one or more paths (it is then called multipath), depending on the topology of the location. In this paper, RFE phenomenon is modeled using only the direct path. It is also supposed in the simulation part that the repeater includes three functions which are a controllable amplification, delay and Additive Gaussian White Noise (AWGN). The channel parameters are chosen to be a constant delay and variable attenuation (other 

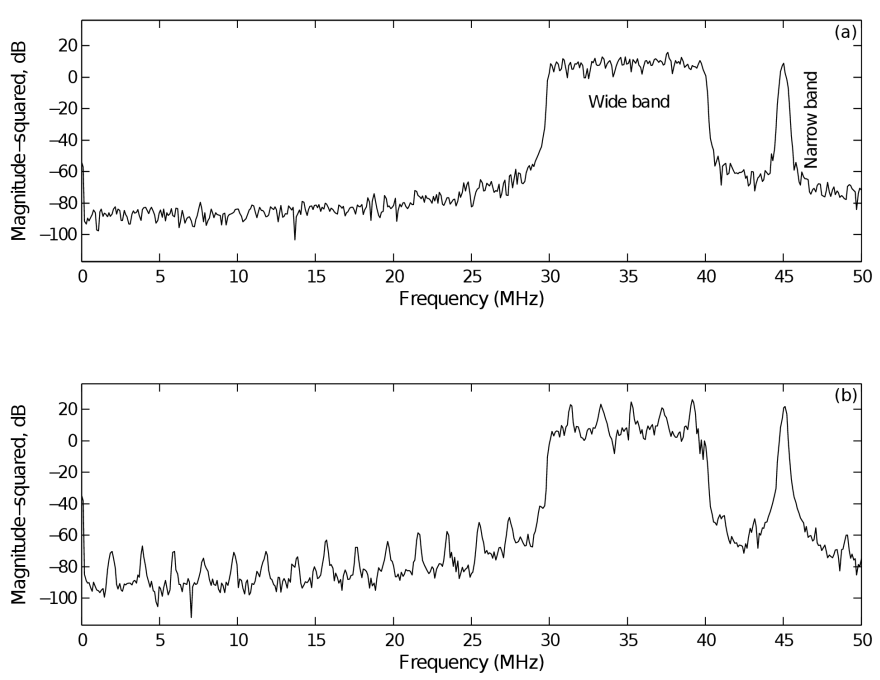

Fig. 1. a) Spectrum of wide band signal (10 $\mathrm{MHz}$ centered on $35 \mathrm{MHz})$ and narrow band (200 $\mathrm{KHz}$ centered on $45 \mathrm{MHz})$. b) Echo is given for gain margin $\Delta=5 \mathrm{~dB}$ and loop delay $\tau=0.5 \mu s$.

parameters as fading are therefore not taken into account) and the propagation delay in wires is supposed negligible.

The article is organized as follows: In section II, mathematical equations modeling the system are presented and the relationships between the power in the repeater and the system parameters as the repeater gain, repeater delay, channel attention are stated. These theoretical results are consistent with findings from the simulation presented in section III. Simulink tool is used to realize the closed-loop system and results are analyzed using Matlab. Finally, as a conclusion, the main results are summarized and discussed.

\section{THEORY}

Taking into account the set of assumptions previously mentioned, a system composed of a repeater, two antennas and a channel is simplified, as shown in Fig. 2. The closed

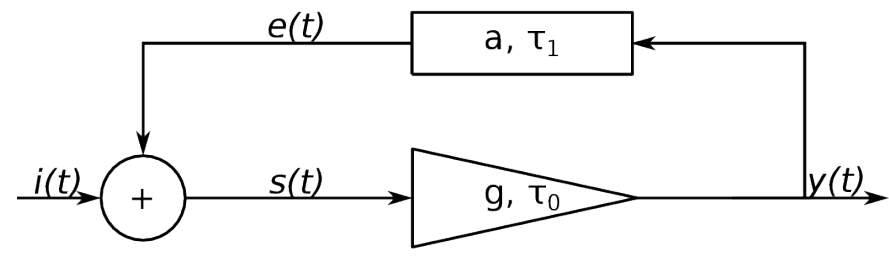

Fig. 2. Radiofrequency coupling, considering only the direct echo path.

loop of the system is expressed by the following equations :

$$
\begin{aligned}
& s(t)=i(t)+e(t), \\
& e(t)=a y\left(t-\tau_{1}\right), \\
& y(t)=g s\left(t-\tau_{0}\right),
\end{aligned}
$$

where $i(t)$ is the incident signal, $y(t)$, the transmitted signal and $e(t)$, the feedback signal (echo). $g$ and $\tau_{0}$ are respectively the repeater gain and the repeater delay, while $a$ and $\tau_{1}$ are respectively the channel attenuation and the channel delay.
System (1) can be expressed such as

$$
y(t)=g \cdot i\left(t-\tau_{0}\right)+g a \cdot y\left(t-\tau_{0}-\tau_{1}\right) .
$$

Eq. (2) is a recursive equation, which can be expressed by $y(t), y\left(t-\left(\tau_{0}+\tau_{1}\right)\right), s\left(t-2\left(\tau_{0}+\tau_{1}\right)\right), \ldots, s\left(t-l\left(\tau_{0}-\right.\right.$ $\left.\left.\tau_{1}\right)\right), s\left(t-(l+1)\left(\tau_{0}-\tau_{1}\right)\right), s\left(t-(l+2)\left(\tau_{0}-\tau_{1}\right)\right)$, while $l$ is the number of secondary echoes. Introducing

$$
\left\{\begin{array}{l}
g \cdot a=\delta \\
\tau_{0}+\tau_{1}=\tau
\end{array}\right.
$$

eq. (2) can be expressed by the following sum

$$
\begin{aligned}
y(t)= & g i\left(t-\tau_{0}\right)+ \\
& g\left[\sum_{n=1}^{l+1}\left(\delta^{n} i\left(t-n \tau-\tau_{0}\right)\right]+\right. \\
& \delta^{l+2} y(t-(l+2) \tau),
\end{aligned}
$$

which can be reduced to its simplest expression, so that

$$
y(t)=g\left[\sum_{n=0}^{l+1} \delta^{n} i\left(t-\left(n \tau+\tau_{0}\right)\right)\right]+\delta^{l+2} y(t-(l+2) \tau) .
$$

Using Fourier Transformation, eq. (4) leads to

$$
\mathcal{Y}(\omega)=\delta^{l+2} e^{-j \omega(l+2) \tau} \mathcal{Y}(\omega)+g \mathcal{I}(\omega) \sum_{n=0}^{l+1} \delta^{n} e^{-j \omega\left(n \tau+\tau_{0}\right)},
$$

where $\mathcal{Y}(\omega)$ and $\mathcal{I}(\omega)$ are the Fourier Transformation of $y(t)$ and $i(t)$, respectively. The transfert function is defined as

$$
H(\omega)=\frac{\mathcal{Y}(\omega)}{\mathcal{I}(\omega)} .
$$

From eq. (5), $H(\omega)$ can be written such as

$$
\mathcal{H}(\omega)=\frac{g \sum_{n=0}^{l+1} \delta^{n} e^{-j \omega\left(n \tau+\tau_{0}\right)}}{1-\delta^{l+2} e^{-j \omega \tau(l+2)}} .
$$

When the repeater steady state is reached, $l$ tends towards infinity. Let $\mathcal{H}_{\infty}$ be this limit. Then, from equation (7), the system transfer function leads to

$$
\mathcal{H}_{\infty}(\omega)=g \frac{e^{j \omega(\tau+\tau 0)}}{-\delta+e^{j \omega \tau}} .
$$

Thus, the power $p$ of the system can be written so that

$$
p(\omega)=\left|\mathcal{H}_{\infty}(\omega)\right|^{2}=\frac{g^{2}}{\delta^{2}-2 \delta \cos (\omega \tau)+1} .
$$

Let us define in $d B$ the following parameters :

$$
\begin{array}{lll}
\text { Power } & : & P=10 \log (p) \\
\text { Gain } & : & G=20 \log (g) \\
\text { Attenuation } & : & A=20 \log (a) \\
\text { Gain margin } & : & \Delta=20 \log (\delta)=G+A .
\end{array}
$$

Using these notations with eq. (9), the power in $\mathrm{dB}$ is given by

$$
P(\omega)=G-10 \log \left(\delta^{2}-2 \delta \cos (\omega \tau)+1\right) .
$$


In practice, most of the variables are expressed in $\mathrm{dB}$, thus

$$
P(\omega)=G-10 \log \left(10^{\Delta / 10}-2 \cdot 10^{\Delta / 20} \cos (\omega \tau)+1\right) .
$$

It is noted that all variables are real such that $G \in \mathbb{R}^{+}, A \in$ $\mathbb{R}^{-}, \Delta \in \mathbb{R}$. Note that in the case of strong echo, corresponding to the fact that gain margin tends towards zero or becomes positive, eq. (12) tends asymptotically to

$$
P(\omega) \approx G-3-10 \log (1-\cos (\omega \tau)) .
$$

Therefore, power value $P$ can theoretically tend to infinity. In the case of weak echo, corresponding to the fact that gain margin is negative and tends towards minus infinity, eq. (12) tends asymptotically to

$$
P(\omega) \approx G
$$

It means that power is constant when sweeping frequency, as illustrated in Fig. 3.
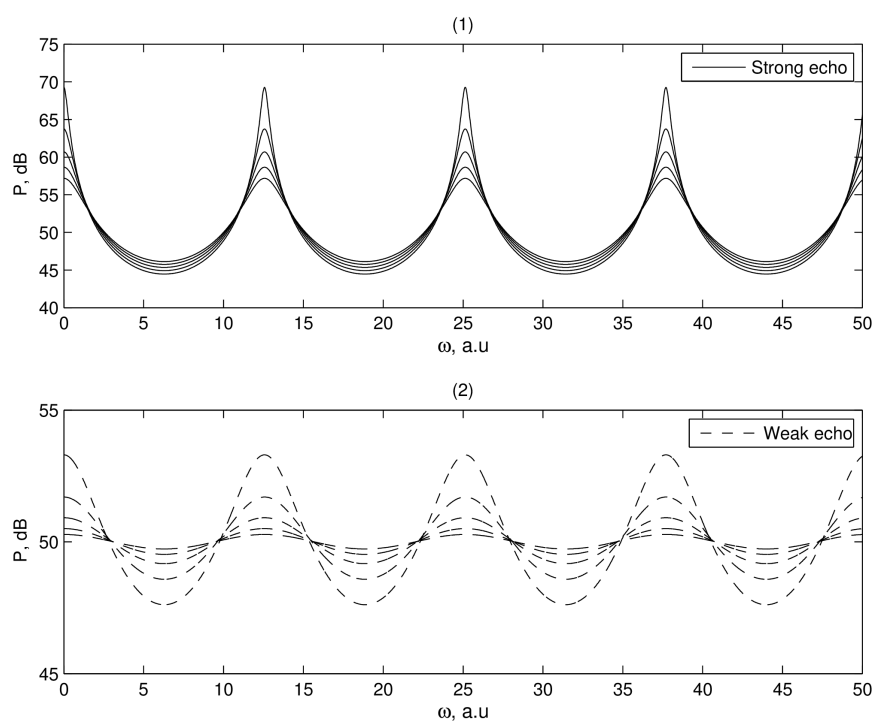

Fig. 3. Representation of power with strong (top figure) and weak (bottom figure) echo for $G=50 \mathrm{~dB}$ and $\tau=0.5$ a.u. Top figure: $\Delta=$ $[-5,-4,-3,-2,-1] \mathrm{dB}$. Bottom figure: $\Delta=[-10,-15,-20,-25,-30]$ $\mathrm{dB}$.

Now, let $R$ be the magnitude of these ripples, defined as the difference between the maximum of power $\left(P_{\max }\right)$ and minimum of power $\left(P_{\min }\right)$. From eq $(12), P_{\max }$ and $P_{\min }$ can be written such as

$$
\begin{aligned}
& P_{\text {max }}(\Delta)=G-10 \log \left(10^{\Delta / 10}-2 \cdot 10^{\Delta / 20}+1\right), \\
& P_{\text {min }}(\Delta)=G-10 \log \left(10^{\Delta / 10}+2 \cdot 10^{\Delta / 20}+1\right),
\end{aligned}
$$

yielding

$$
R(\Delta)=20 \log \left|\frac{10^{\Delta / 20}+1}{10^{\Delta / 20}-1}\right|,
$$

which is indeed only depending on gain margin $\Delta$. When gain margin is high, the ripple magnitude becomes infinitely large, consequently, the repeater receives multiple peaks of power depending on the number of channels in their frequencies.
Nevertheless, a positive gain margin without AGC or RFE cancellation system cannot exist in practice, because electronics circuits may be damaged before reaching zero gain margin. In order to characterize this ripple phenomenon, it is also interesting to determine the delay and the attenuation. $P(\omega)$ is a periodic function whose period $\rho(\tau)$ can be expressed, from eq. (12) such as

$$
\rho(\tau)=\frac{2 \pi}{\tau},
$$

while, attenuation of the channel can be written so that

$$
A(R)=20 \log \left(\frac{10^{R / 20}-1}{10^{R / 20}+1}\right)-G .
$$

From eq. (17) and eq. (16), dualistically, the main parameters of the echo, i.e. $\Delta$ and $\tau$, are given by

$$
\left\{\begin{array}{l}
\Delta(R)=20 \log \left|\frac{10^{R / 20}+1}{10^{R / 20}-1}\right|, \\
\tau(\rho)=\frac{2 \pi}{\rho}
\end{array}\right.
$$

Therefore, as ripple magnitude $R$ and the period $\rho$ can be measured by using frequency sweeping technique, it is possible to measure indirectly $\Delta$ and $\tau$. Therefore it is possible to characterize RFE, which is presented in the next section.

\section{Simulation}

In this part, the theoretical results are compared to simulated ones by using Simulink, where a signal generator block provides a sinusoidal signal with AWGN and both signal and noise parameters are independently adjustable. The echo creator is composed of a repeater (gain, delay, noise) and a channel (attenuation, delay) model in closed-loop configuration. Thus, the first echo is added to the principal signal after $\tau$ delay; the $l^{\text {th }}$ echo is added after $l \tau$ delay as expressed in eq. (4). Echoes are reduced by a $A \mathrm{~dB}$ factor for every passing through the loop. Hence, the first echo is reduced by $A \mathrm{~dB}$ and the $l^{\text {th }}$ echo is reduced by $l A \mathrm{~dB}$. A peak detector block is realized by using the Fast Fourier Transform (FFT) in order to calculate the Power Spectral Density (PSD). The peak detector block provides the value of the maximum (in $\mathrm{dB}$ ) for any frequency value, when sweeping. Finally, the result exporter block exports simulation parameters and results to a database.

Unless otherwise specified, the sampling frequency is set to $100 \mathrm{MHz}$. The frequency sweep ranges from $20 \mathrm{MHz}$ to 30 $\mathrm{MHz}$ with a frequency sweep step of $1 \mathrm{KHz}$ and is realized with a cosine signal without any modulation. The channel attenuation is set to $-50 \mathrm{~dB}$. Finally, the other variables are depending on each case. A Gui window is used to control system parameters automatically, sweeping frequency, gain, etc.

Fig. 4 shows an example of sweeping frequency, with a frequency range of $200 \mathrm{KHz}$. It provides a comparison of the maximum of PSD value calculated in theory, in equation (12), 


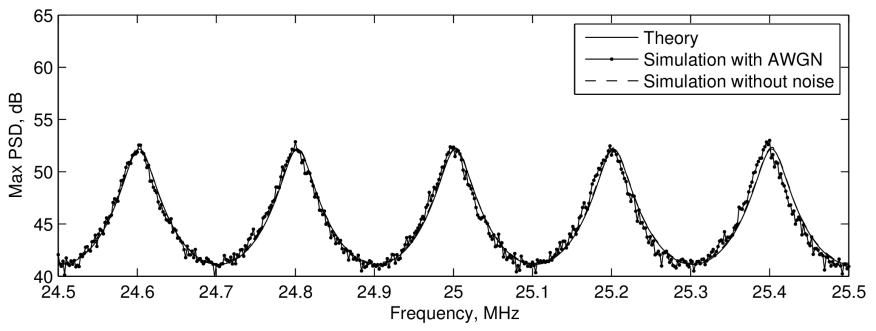

Fig. 4. The maximum of PSD, with $A=-50 \mathrm{~dB}, G=45 \mathrm{~dB}$ ( $\Delta=5$ $\mathrm{dB}), \tau=0.5 \mu \mathrm{s}$, without noise (dashed curve) and with $S N R=30 \mathrm{~dB}$.

and simulation results. A systematic study has been performed for different values of $\Delta$ and $\tau$, showing again a very good match between simulation results and theoretical predictions. It has also been observed that the measures of maximum of PSD are only slightly sensitive to noise, as illustrated in Fig. 4. Concerning the ripple magnitude $R$, a systematic study in function of the gain margin is presented in Fig. 5 where theoretical results and simulation results for different values of AWGN can be compared. As it can been observed, for a high SNR, errors between simulation results and theory (equation 16) are negligeable and even for low SNR (for instance 30 $\mathrm{dB})$, the errors remain very small.

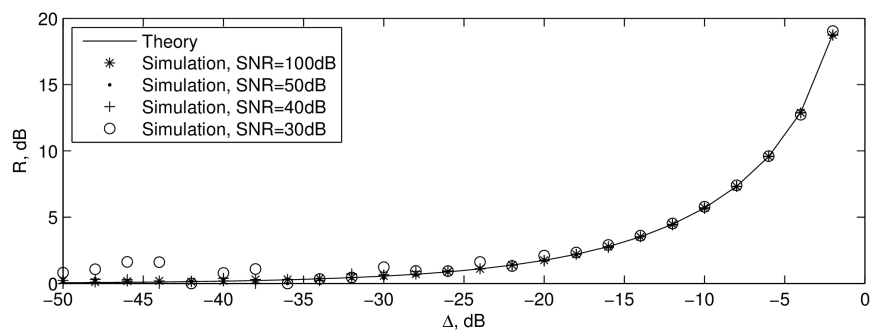

Fig. 5. The ripple magnitude $R$ depending on the gain margin $\Delta$, comparing theory and simulation for different values of SNR.

In equation (19), it is shown that the gain margin $\Delta$ is depending only on the ripple magnitude $R$ and the loop delay $\tau$ is depending only on the period of ripple $\rho$. By using an appropriate system of data acquisition, as digital acquisition using a Field-Programmable Gate Array (FPGA), $R$ and $\tau$ can be estimated using a nonlinear estimating technique as leastsquares [7], Gauss-Newton or Levenberg-Marquardt [8] to cite but a few. Hence $\Delta$ and $\tau$ can be calculated using eq. (19) with the estimated value of $R$ and $\rho$.

In the following, the effect of AWGN, the gain margin and the band width on the Relative Error Estimation (REE) are discussed.

Since it has been demonstrated that ripple magnitude are depending on the gain margin value (eq. 16), estimation precision is naturally depending on the gain margin value which is corrupted by the AWGN. Top part of Fig. 6 shows the REE of the estimation of $\Delta$ depending on the gain margin value for different values of SNR. According to the figure, gain margin is best estimated for SNR values more than 30
$\mathrm{dB}$ and gain margin values between -10 and $-5 \mathrm{~dB}$.
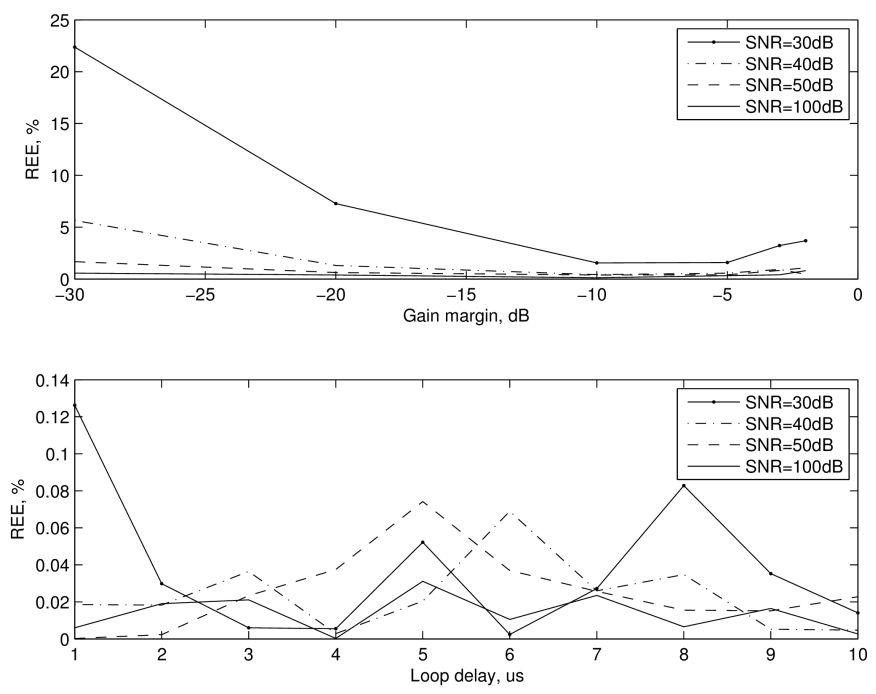

Fig. 6. Gain margin $\Delta$ (top figure) and Loop delay $\tau$ (bottom figure) estimation. Input parameters : $\Delta=10 \mathrm{~dB}$ for $\tau$ estimation, $\tau=5 \mu \mathrm{s}$ for $\Delta$ estimation.

Loop delay estimation is optimal for any SNR value according to the lower part of Fig. 6, as the corresponding REE remains lower than $0.14 \%$, even if the SNR reaches $30 \mathrm{~dB}$.

Sweeping frequency can be a slow operation (with a large frequency range and a small sweeping step). In practice, emitting signals must be in accordance with each country's rules and regulations, this is why the bandwidth effect is studied in this section. Indeed, it is important to reduce to a minimize the range of sweeping.
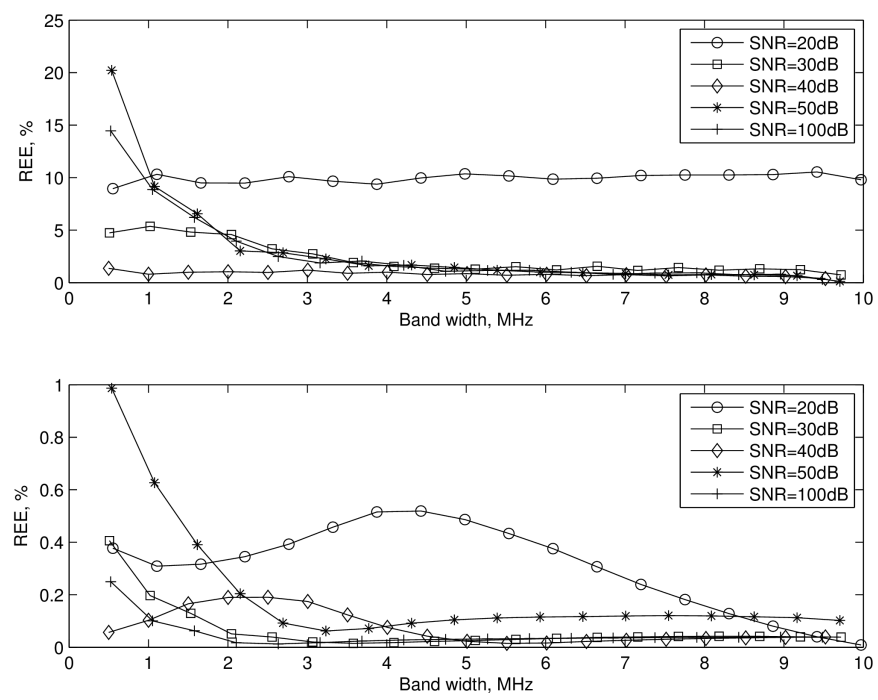

Fig. 7. Gain margin $(\Delta=10 \mathrm{~dB})$ and Loop delay $(\tau=5 \mu s)$ estimation for different values of SNR.

Top part of Fig. 7 shows that REE, when estimating gain margin, is less than $5 \%$ for $S N R>30 \mathrm{~dB}$ and bandwidth 
larger than $2 \mathrm{MHz}$, while bottom part of Fig. 7 shows that REE, when estimating loop delay, is less than $0.5 \%$ for $S N R>30 \mathrm{~dB}$ and bandwidth larger than $2 \mathrm{MHz}$. In all cases, SNR values, which corresponds to the quality of the signal, implies a limitation for an accurate estimation of the parameters characterizing RFE. Simulations have also shown that eq. (19) is satisfied, i.e, echo parameters are well measured.

\section{CONCLUSION}

This paper discussed a method for radio frequency echo detection and measurement by sweeping carrier frequency and by analyzing ripples of the power spectrum density. Mathematical expressions have been established. These expressions have been confirmed by simulation results.

This solution can be used in addition to an echo cancellation system [9] to improve the efficiency and shut down the echo cancellation system during the absence of radio frequency echo in order to economize energy.

In a simplified model of radio frequency echo, the main parameters are the loop delay $(\tau)$ and the gain margin $(\Delta)$. These parameters are calculated in theory and confirmed by simulation. Nonlinear estimation methods have been used in this work. It has also been shown the influence of noise (AWGN) and the range of frequency sweeping on the echo parameter estimation.

This solution could be used to detect the presence of echoes and to trigger alarm after leaving specified value in repeaters, so that it could prevent the signal corrupting and system damage against sudden arrivals of radio frequency echo dues to reflections, climate changes, etc.

Indeed, it has been shown, in simulation part, that the RFE parameters are correctly estimated (less than 5\%) in noisy environment $\mathrm{SNR} \approx 30 \mathrm{~dB}$ and a range less than $2 \mathrm{MHz}$ with $1 \mathrm{KHz}$ of sweeping step. Under these conditions, radio frequency echo parameters could be used to cancel the echo by creating a feedback and learning loop. It would be possible to enhance these results by increasing system performance as noise reduction in repeater, frequency sampling in digital part, estimating method and so on. As a perspective of future work, this study could be extended to the case of multipath or modulated signals.

\section{ACKNOWLEDGMENT}

The authors would like to acknowledge support from the Conseil Régional de Bourgogne (Regional Council of Burgundy) in the framework of the PARI program and ANRT agency (French National Agency for Research and Development) in the framework of CIFRE program.

\section{REFERENCES}

[1] M. Sondhi and D. Berkley, "Silencing echoes on the telephone network," Proceedings of the IEEE, vol. 68, pp. 948 - 963, aug. 1980.

[2] R. Braithwaite, S. Carichner, and M. Cope, "Echo cancellation for a wide bandwidth mixed-mode wcdma/gsm repeater with digital sub-band filtering," in Vehicular Technology Conference, 2009. VTC Spring 2009. IEEE 69th, pp. $1-5$, april 2009.

[3] J. kyu Hong, Y. woo Suh, J. yong Choi, and J.-S. Seo, "Echo canceller for on-channel repeaters in t-dmb system," in Advanced Communication Technology, 2008. ICACT 2008. 10th International Conference on, vol. 3, pp. $1735-1738$, feb. 2008

[4] E. Hänsler and G. Schmidt, Acoustic Echo and Noise Control: A Practical Approach. Adaptive and Learning Systems for Signal Processing, Communications, and Control, Wiley-Interscience, 2004.

[5] A. Guerin, G. Faucon, and R. Le Bouquin-Jeannes, "Nonlinear acoustic echo cancellation based on volterra filters," Speech and Audio Processing, IEEE Transactions on, vol. 11, pp. 672 - 683, nov. 2003.

[6] X. Lagrange, P. Godlewski, and S. Tabbane, Réseaux GSM Des principes à la norme. Hermès - Lavoisier, 2000.

[7] D. Marquardt, "An algorithm for least-squares estimation of nonlinear parameters," Journal of the Society for Industrial and Applied Mathematics, pp. 431-441, 1963.

[8] H. Gavin, The Levenberg-Marquardt method for nonlinear least squares curve-fitting problems. Department of Civil and Environmental Engineering, Duke University, September 282011.

[9] M. Lee, B. Keum, H. S. Lee, and J.-W. Kim, "A radio repeater interference cancellation model for mobile communication systems," in Wireless and Mobile Communications, 2008. ICWMC '08. The Fourth International Conference on, pp. 376-381, 27 2008-aug. 12008. 